\title{
Complex $\mathrm{N}$-glycans or core 1-derived O-glycans are not required for the expression of stage-specific antigens SSEA-1, SSEA-3, SSEA-4, or Le ${ }^{\mathrm{Y}}$ in the preimplantation mouse embryo
}

\author{
Suzannah A Williams* and Pamela Stanley \\ Department of Cell Biology, Albert Einstein College of Medicine, New York, NY, 10461, U.S.A
}

\begin{abstract}
The glycan epitopes termed stage-specific embryonic antigens (SSEA) occur on glycoproteins and glycolipids in mammals. However, it is not known whether these epitopes are attached to N- or Oglycans on glycoproteins and/or on glycolipids in the developing mouse embryo. In this paper the expression of the antigens SSEA-1, SSEA-3, SSEA-4 and Le ${ }^{\mathrm{Y}}$ was examined on ovulated eggs, early embryos and blastocysts lacking either complex and hybrid $\mathrm{N}$-glycans or core-1 derived O-glycans. In all cases, antigen expression determined by fluorescence microscopy of bound monoclonal antibodies to embryos at the stage of development of maximal expression, was similar in mutant and control embryos. Thus, none of these developmental antigens are expressed solely on either complex $\mathrm{N}$ - or core 1-derived O-glycans attached to glycoproteins in the preimplantation mouse embryo. Furthermore, neither of these classes of glycan is essential for the expression of SSEA-1, SSEA-3, SSEA-4 or $\mathrm{Le}^{\mathrm{Y}}$ on mouse embryos.
\end{abstract}

\section{Keywords}

N-glycans; O-glycans; Mgat 1; T-synthase; preimplantation mouse embryos; SSEA; immunofluorescence microscopy

\section{Introduction}

Stage-specific embryonic antigens (SSEA) are present on eggs and preimplantation embryos at specific stages of early mouse development [1-5]. The epitope of a number of these antigens consists solely of sugar residues. SSEA-1, also known as the Lewis $\mathrm{X}$ antigen $\left(\mathrm{Le}^{\mathrm{X}}\right)$, is Gal $\beta 1,4[$ Fuc $\alpha 1,3]$ GlcNAc $\beta 1,3$ Gal $\beta 1-R$, SSEA-3 is Gal $\beta 1,3$ GalNAc $\beta 1,3$ Gal $\alpha 1,4$ Gal $\beta 1,4$ Glc $\beta 1-R$, SSEA-4 is sialylated SSEA-3 (NeuAc $\alpha 2,3 \mathrm{Gal} \beta 1,3 \mathrm{GalNAc} \beta 1,3 \mathrm{Gal} \alpha 1,4 \mathrm{Gal} \beta 1,4 \mathrm{Glc} \beta 1-\mathrm{R})$ and the Lewis $\mathrm{Y}$ antigen $\left(\mathrm{Le}^{\mathrm{Y}}\right)$ is Fuc $\alpha 1,2 \mathrm{Gal} \beta 1,4[\mathrm{Fuc} \alpha 1,3] \mathrm{GlcNAc} \beta 1,3 \mathrm{Gal}-\mathrm{R}$. Monoclonal antibodies to these antigens were raised about 30 years ago [2].

The first developmentally-regulated SSEA was identified following immunization of mice with F9 embryonal carcinoma cells [1]. The IgM monoclonal antibody (MAb) bound to F9 cells and to mouse embryos, and the antigen it recognized was termed SSEA-1. SSEA-1 is not detected on mouse embryos until the 8-cell compacted stage [1,6,7]. Expression continues through morula and early blastocyst but is lost by the late blastocyst stage [1,7]. The epitope recognized by the SSEA- 1 MAb is Gal $\beta 1,4[\mathrm{Fuc} \alpha 1,3] \mathrm{GlcNAc} \beta 1$, as determined by competitive

\footnotetext{
*Current address: Department of Physiology, Anatomy and Genetics, University of Oxford, South Parks Road, Oxford, OX1 3QX, Great Britain.
} 
binding assays using isolated oligosaccharides [8], and by structural analyses of glycolipids [9]. Based on the highly regulated expression pattern of SSEA-1 during blastogenesis, it was proposed that this cell surface antigen might be involved in compaction of the embryo. In fact, compaction was inhibited when 4-8-cell mouse embryos were incubated with oligosaccharides carrying the SSEA-1 determinant [10,11]. However, when the $\alpha 1,3$ fucosyltransferase Fut 9 which adds the fucose of the SSEA- 1 epitope was inactivated by targeted mutation, compaction occurred normally in $\mathrm{Fut}^{-{ }^{-1-}}$ mouse embryos, despite a dramatic reduction in the SSEA-1 determinant on late 8-cell embryos [12]. This experiment showed that Fut9 was largely, if not completely, responsible for generating SSEA-1 in mouse embryos, and that the expression of SSEA-1 is not essential for compaction or for mouse development. Nevertheless, expression of SSEA-1 is highly regulated and may have a functional role during blastogenesis. Thus, it is of interest to know whether this antigen is expressed on $\mathrm{N}$-glycans and/or core 1-derived Oglycans of the embryo. Both these glycans are found only on glycoproteins. However, the SSEA-1 determinant, which occurs at the non-reducing terminus of a glycan, occurs on a wide range of glycolipids and glycoproteins in mammalian cells and tissues [3,13-20].

SSEA-3 was identified following immunization of rats with 4-8 cell mouse embryos [2] and was present on glycolipids of human teratocarcinoma cells [21,22]. Immunoprecipitation of SSEA-3 from mouse embryos revealed numerous proteins by acrylamide gel electrophoresis of the immunoprecipitate [23]. In embryonal carcinoma cells expressing this antigen, tunicamycin treatment markedly decreased proteins immunoprecipitated by the SSEA-3 MAb, indicating that $\mathrm{N}$-glycans express this epitope. SSEA-4 was present on globo-series glycolipids and glycoproteins of teratocarcinoma cells [21,23]. SSEA-3 and SSEA-4 epitopes are thought to be expressed during oogenesis since they are present on mouse eggs and early embryos, and persist until the late blastocyst stage [24].

The $\mathrm{Le}^{\mathrm{Y}}$ epitope was first identified on human red blood cells and monoclonal antibodies were subsequently developed $[7,25]$. $\mathrm{Le}^{\mathrm{Y}}$ is not detected on 2-4 cell mouse embryos but is expressed at the morula stage and on blastocysts [7]. Interestingly, $\mathrm{MAb}$ against $\mathrm{Le}^{\mathrm{Y}}$ inhibits mouse blastocyst implantation $[26,27]$. The $\mathrm{Le}^{\mathrm{Y}}$ epitope is expressed on glycolipids and glycoproteins in mammalian cells and tissues.

Each of the glycan epitopes described above is expressed on mouse preimplantation embryos but it is not known if the epitopes are carried on N- or O-glycans of glycoproteins and/or on glycolipids. To begin to investigate this question we developed female mice that ovulate eggs lacking T-synthase ( $T$-syn), the core $1 \beta 1$,3galactosyltransferase necessary for the synthesis of core 1-derived O-glycans [26,27]. By crossing these females with $T$-syn ${ }^{+/-}$males, $T$-syn ${ }^{-1-}$ embryos are generated. These embryos do not express core 1 or core 2 O-GalNAc glycans (Fig. 1) and were used in this work to determine if these glycans are essential for the synthesis of SSEA-1, SSEA-3, SSEA-4, or $\mathrm{Le}^{\mathrm{Y}}$ during mouse embryogenesis. We have also generated female mice that ovulate eggs lacking GlcNAcT-I (Mgat1), the glycosyltransferase responsible for converting oligomannose $\mathrm{N}$-glycans to complex or hybrid N-glycans (Fig. 1) [28]. By crossing to $\mathrm{Mgatl}^{+-}$males, maternal and zygotic $\mathrm{Mgatl}^{-/-}$embryos are obtained. These mutant embryos do not express complex or hybrid $\mathrm{N}$-glycans on their glycoproteins and were used to ask if these $\mathrm{N}$-glycans are essential for the expression of the developmentally-regulated glycan antigens described above.

In this paper we use indirect fluorescence microscopy to compare monoclonal antibody binding to SSEAs of preimplantation mouse embryos that lack complex and hybrid $\mathrm{N}$-glycans or core 1-derived O-glycans. We show that embryos lacking complex N- or core 1-derived O-glycans express each of the four SSEAs similarly to control embryos. Therefore, neither of these classes of glycans is essential for the expression of any of the four developmental antigens. 


\section{Materials and Methods}

\section{Mice}

To generate preimplantation mouse embryos that were maternal and zygotic null for the glycosyltransferases T-synthase or GlcNAcT-I, floxed T-syn or Mgat1 genes were deleted in oocytes. Females of mixed genetic background (predominantly C57Bl/6 and 129SvJ), homozygous for $T$-syn floxed alleles $\left(T-s y n^{F}\right)$ and carrying a Cre recombinase transgene under the control of the oocyte-specific ZP3 promoter (T-syn ${ }^{\mathrm{F} / \mathrm{F}}: \mathrm{ZP} 3 C r e$ females), ovulate oocytes lacking T-synthase [29]. ZP3 is expressed exclusively in oocytes at the primary follicle stage of development [30]. The ZP3Cre transgenic strain was originally obtained from Dr. Jamey Marth [28]. To obtain embryos lacking core 1-derived O-glycans, $T$-syn ${ }^{\mathrm{F} / \mathrm{F}}: \mathrm{ZP} 3 \mathrm{Cre}$ females were crossed with $T-$ syn $^{+/-}$males producing both $T-$ syn $^{+/-}$and $T-$ syn $^{-/-}$embryos in a 1:1 ratio. To generate additional control embryos, $T-s y{ }^{\mathrm{F} / \mathrm{F}}$ females lacking the $\mathrm{ZP} 3 \mathrm{Cre}$ transgene were crossed with either $T$-syn ${ }^{+/+}$or $T$-syn ${ }^{+/-}$males. Mice heterozygous for either T-syn or Mgat1 have no apparent phenotype. The respective floxed genes behave indistinguishably from wild type, and therefore floxed alleles of eggs or embryos are designated ' + '. No developmental effects of expressing the ZP3Cre transgene have been observed in these models [28,29].

The same breeding strategy was used to obtain embryos lacking complex N-glycans. Mgat $^{\mathrm{F} / \mathrm{F}}: \mathrm{ZP} 3 \mathrm{Cre}$ females [28] were mated to $\mathrm{Mgat1}^{+/-}$males and for additional control embryos, gat $^{\mathrm{F} / \mathrm{F}}$ females were mated to ggat $^{+/-}$or Mgat $^{+/+}$males. Mgat ${ }^{\mathrm{F} / \mathrm{F}}: \mathrm{ZP} 3 \mathrm{Cre}$ females were also generated from ZP3Cre strain 93 kindly provided by Dr. Barbara Knowles [31]. Genotyping of parental mice was performed by PCR of tail genomic DNA as previously described $[28,29]$.

\section{Egg and embryo collection and preparation}

To generate mouse embryos at specific stages of development, females were joined with males and mating was confirmed in the morning by the presence of a vaginal plug. Assuming coitus had taken place at midnight, 2-cell embryos were collected at 1.25 days post coitum (pc), 4-8 cell embryos were collected at 2-2.5 days pc, 8-cell compacted embryos were collected at $\sim 2.75$ days pc, and blastocysts were collected at 3.5-4 days pc.

Embryos were collected into KSOM+AA culture medium (Specialty Media, Phillipsburg, NJ) that had been equilibrated overnight at $37^{\circ} \mathrm{C}$ in an incubator with $95 \% \mathrm{CO}_{2}$ and $5 \%$ air. Preblastocyst stage embryos were flushed from oviducts, and blastocysts were flushed from uterine horns using equilibrated medium. Embryos from individual females were examined or, embryos from several females were pooled, and split into groups for sequential lectin and monoclonal antibody (MAb) binding.

To obtain ovulated eggs, females were induced to ovulate by intraperitoneal administration of $5 \mathrm{IU}$ pregnant mare's serum gonadotrophin (Calbiochem, La Jolla, CA) followed by $5 \mathrm{IU}$ human chorionic gonadotrophin (hCG; Sigma, St. Louis, Mo.) 46-48 hours later. Eggs ovulated $\sim 14$ hours later and were collected from oviducts 14-16 hours post hCG. Oviducts containing ovulated eggs were dissected in M2 medium (Specialty Media) that had been equilibrated overnight at $37^{\circ} \mathrm{C}$ in an incubator with $95 \% \mathrm{CO}_{2}$ and $5 \%$ air. Cumulus masses were released from the oviducts into M2 medium containing $0.3 \mathrm{mg} / \mathrm{ml}$ hyaluronidase (Sigma) and protease inhibitors (Roche, Indianapolis, IN). The eggs were incubated in the hyaluronidase for $\sim 5$ min to remove cumulus cells and then washed in M2 medium.

The zona pellucida was removed from eggs and embryos by brief immersion in Tyrodes acid and washing in $\mathrm{M} 2$ or KSOM+AA medium respectively before fixation in $2 \%$ paraformaldehyde for $1 \mathrm{hr}$ at room temperature. Eggs and embryos were then washed in phosphate buffered saline containing 3\% bovine serum albumin (PBS/BSA) by transferring 
through 3 sequential droplets under mineral oil. Consecutive washing through three droplets under mineral oil was used for all egg/embryo medium transfers from the initial blocking stage onwards. If embryos were to be stored before further antibody incubation, as was often the case for negative control embryos, they were stored at $4^{\circ} \mathrm{C}$ in PBS/BSA. Embryos of 2-4 cells were included as negative controls for SSEA-1 and $\mathrm{Le}^{\mathrm{Y}}$ expression, and late stage blastocysts were included as negative controls for SSEA-3 and SSEA-4 expression [7,24].

\section{Lectin binding}

Eggs and embryos were phenotyped based on their lectin binding properties [29,32]. To block non-specific lectin binding sites, eggs and embryos were incubated in PBS/BSA for $1 \mathrm{hr}$ at room temperature before incubation in lectin. Complex and hybrid $\mathrm{N}$-glycans were detected by incubation of eggs and embryos in $20 \mu \mathrm{g} / \mathrm{ml}$ Phaseolus vulgaris leukoagglutinin conjugated to fluorescein isothiocyanate (L-PHA-FITC; Vector Labs, Burlingame, CA) for $1 \mathrm{hr}$ at room temperature. Core 1-derived O-glycans were detected by incubation of embryos with $20 \mu \mathrm{g}$ / $\mathrm{ml}$ peanut agglutinin conjugated to FITC (PNA-FITC; Vector Labs) for $1 \mathrm{hr}$ at room temperature. All steps from the start of lectin incubation with the exception of egg/embryo transfer were performed in the dark to minimize photobleaching.

\section{Antibody binding}

Following incubation in lectin, eggs and embryos were washed in PBS/BSA by 3 drop transfer and incubated in MAb for $1 \mathrm{hr}$ at room temperature. After primary antibody, eggs and embryos were washed, transferred to the appropriate secondary antibody and incubated for $1 \mathrm{hr}$ at room temperature. Eggs and embryos were then washed and photographed in the last of the 3 droplets.

SSEA-1/Le ${ }^{\mathrm{X}}$ was detected using the SSEA-1 MAb in conditioned medium diluted at 1/100 prepared from hybridoma cells kindly provided by Dr. Barbara Knowles [1]. After washing in PBS/BSA embryos were incubated in tetramethylrhodamine isothiocyanate (TRITC)conjugated goat anti-mouse IgM at 1/100 in PBS/BSA (Southern Biotech). Eggs and embryos were examined for SSEA-3 and SSEA-4 expression using MAbs [21] in undiluted hybridoma cell conditioned medium. The monoclonal antibodies to SSEA3 and SSEA4 developed by B. B. Knowles and D. Solter were obtained from the Developmental Studies Hybridoma Bank developed under the auspices of the NICHD and maintained by The University of Iowa, Department of Biological Sciences, Iowa City, IA 52242 (MC-631 for SSEA-3 and MC-813-70 for SSEA-4). After washing, SSEA-4 MAb binding was detected using rhodamine-conjugated donkey anti-mouse IgG at 1/100 in PBS/BSA (Jackson Labs), and SSEA-3 MAb binding was detected using TRITC-conjugated goat anti-rat IgG at 1/100 (Jackson Labs). $\mathrm{Le}^{\mathrm{Y}}$ expression was detected using clone F3 monoclonal antibody at 1/50 dilution in PBS/BSA (Calbiochem), washing in PBS/BSA and incubation in TRITC-conjugated goat anti-mouse IgM at 1/100 in PBS/BSA (Southern Biotech).

\section{Fluorescence microscopy}

Fluorescence microscopy was performed with an inverted Olympus fluorescence microscope using filters for the detection of fluorescein or rhodamine. Control and mutant eggs and embryos were photographed in the last wash droplet under oil with the phase pictures and different exposures taken in exactly the same order to ensure that photobleaching was minimal and equivalent. Within each experiment, the phase picture was taken using controls first, followed by increasing exposures ( 0.125 up to $2 \mathrm{sec}$ ) of FITC fluorescence and then by increasing exposures $(0.125$ up to $2 \mathrm{sec})$ of TRITC fluorescence, with the same sequence then repeated with mutant embryos. The exposures shown in the figures were selected as the level of fluorescence that allowed embryos with no binding to be just visible - generally a $1 \mathrm{sec}$ exposure. 


\section{Results}

\section{Identification of control and mutant eggs and embryos}

In order to directly compare control and mutant eggs and embryos for developmental antigen expression, they were obtained from several control and mutant females for analysis in the same experiment. Embryos at a stage that should not bind the MAb were added to each group. Control embryos are distinguished from $T$-syn mutant embryos by their different abilities to bind PNA (Fig. 1), as previously described for ovulated eggs [29]. PNA binding was strong in most control embryos (Fig. 2B). However, 2-4 cell control embryos did not bind PNA as well as embryos at later stages (Fig. 2B). This may be because expression of T-synthase is reduced at the $2-4$ cell stage, or due to the addition of sialic acid to core 1 or of GlcNAc to form core 2 O-glycans (Fig. 1). Importantly however, late 8-cell compacted wild type embryos bound PNA well (Fig. 2B), whereas $T$-syn mutant embryos at the same stage did not bind PNA (Fig. $2 \mathrm{E})$. The fact that heterozygous compacted embryos present with mutant embryos in Fig. 2E did not bind PNA as well as control compacted embryos in Fig. 2B most likely reflects a delay in paternal $T$-syn gene activation. Thus, heterozygous embryos derived from mutant females receive their active $T$-syn gene following fertilization, and translation from paternal transcripts may not occur until the 4- to 8-cell or the morula stage as was shown for $\beta$-catenin and Ecadherin, respectively [33]. Nevertheless, 8 -cell compacted $T$-syn ${ }^{-1-}$ mutant embryos were readily identified by their lack of PNA binding (circled in Fig. 2E). Wild type eggs and embryos that express Mgat1 synthesize complex N-glycans that bind the lectin L-PHA [28,34] (Fig. 1). It can be seen in Fig. 3 that mixed wild type and heterozygous control embryos bound L-PHA similarly (Fig. 3B) and mutant embryos were readily identified by their reduced L-PHA binding (circled in Fig. 3E).

\section{Expression of SSEA-1 in mouse embryos lacking core 1-derived O-glycans}

The embryos shown in Fig. 2A and 2D were compared for binding of the MAb against SSEA-1. Embryos collected at the 2-4 cell stage when SSEA-1 is not expressed, were mixed with 8cell control and mutant compacted embryos. It can be seen in Fig. $2 \mathrm{C}, 2 \mathrm{C}^{\prime}, 2 \mathrm{~F}$ and $2 \mathrm{~F}^{\prime}$ (dashed arrows) that 2-4-cell embryos did not express SSEA-1 and provided appropriate negative controls for background levels of SSEA-1 MAb binding. By contrast, 8-cell compacted control embryos bound SSEA-1 very well (Fig. $2 \mathrm{C}$ and $2 \mathrm{C}^{\prime}$ ). Since the expression of SSEA-1 is tightly regulated, those embryos that exhibited less binding of SSEA-1 or PNA may be at a slightly earlier or later stage of development. T-syn mutant 8-cell compacted embryos, identified on the basis of their lack of PNA binding (circled in Fig. 2E and 2F), also bound the SSEA-1 MAb well (Fig. 2F and 2F', solid arrows). SSEA-1 MAb binding of PNA-negative embryos (Fig. 2F and $2 \mathrm{~F}^{\prime}$ ) was similar to wild type embryos (Fig. $2 \mathrm{C}$ and $2 \mathrm{C}^{\prime}$ ). These images are representative of 20 compacted embryos collected from mutant females. Thus, core 1-derived O-glycans are not essential for the expression of SSEA-1. Embryos lacking core 1-derived O-glycans must therefore express the SSEA-1 epitope predominantly on N-glycans of glycoproteins and/or on glycolipids.

\section{Expression of SSEA-1 in mouse embryos lacking complex and hybrid N-glycans}

Embryos lacking complex and hybrid N-glycans, identified by their lack of L-PHA binding (circled in Fig. 3E, solid arrows in Fig. 3E'), were compared to control embryos at the 8-cell compacted stage for binding of the SSEA-1 MAb. As observed in Fig. 2, control embryos bound the SSEA-1 MAb well at the 8-cell compacted stage but not at the 2-4 cell stage (Fig. $3 \mathrm{C}$ and $3 \mathrm{C}^{\prime}$, dashed arrows). Mgatl mutant embryos also bound the SSEA-1 MAb well (circled in Fig. $3 \mathrm{E}$ and $3 \mathrm{~F}$, solid arrows in $3 \mathrm{E}^{\prime}$ and $3 \mathrm{~F}^{\prime}$ ). The smaller 8-cell compacted mutant embryo had somewhat aberrant morphology which was to be expected in embryos generated from $\mathrm{Mgatl}^{-1-}$ eggs of which $\sim 50 \%$ are retarded in development [28]. Overall, in comparisons of 12 compacted 8 -cell embryos from control and 8 from mutant females, SSEA-1 MAb binding 
was similar between control and mutant. Thus, complex or hybrid N-glycans are not essential for the expression of the SSEA-1.

\section{Expression of SSEA-3 in mouse embryos lacking core 1-derived O-glycans}

SSEA-3 is present on eggs and preimplantation embryos until the blastocyst stage [23,24]. To determine if core 1-derived O-glycans are substantial carriers of the SSEA-3 epitope, 4-8 cell embryos were collected. Control embryos and blastocysts were PNA-positive (Fig. 4B and 4B ') while $T$-syn ${ }^{-/}$embryos from $T-s y n^{\mathrm{F} / \mathrm{F}}$ :ZP3Cre females were PNA-negative (circled in Fig. 4E and 4F). Control embryos at the 4-8 cell stage bound the SSEA-3 MAb well but fluorescence was greatly reduced at the late blastocyst stage which constituted the negative control for the SSEA-3 MAb (Fig. 4C and 4F, dashed arrows). T-syn mutant embryos (circled in Fig. 4E and 4F) bound the SSEA-3 MAb similarly to control embryos as shown in Fig. 4F and 4F' (solid arrows). T-syn mutant eggs also bound the SSEA-3 MAb equivalently to control eggs (not shown). Thus SSEA-3 is not expressed at observable levels on core 1-derived O-glycans in eggs or early embryos, suggesting that SSEA-3 is predominantly expressed either on complex $\mathrm{N}$-glycans and/or on glycolipids.

\section{Expression of SSEA-3 in mouse eggs and embryos lacking complex and hybrid N-glycans}

To investigate a role for complex N-glycans in the expression of SSEA-3 during embryogenesis, eggs and embryos were obtained from Mgat $^{\mathrm{F} / \mathrm{F}}: \mathrm{ZP} 3 \mathrm{Cre}$ females mated to Mgat $^{+/-}$males. Since both SSEA-3 and SSEA-4 are expressed on ovulated eggs and embryos until the blastocysts stage [24], ggat $^{-1-}$ ovulated eggs were used for analyzing the expression of these two antigens because Mgat1 mutant females have low fertility. Eggs could be readily collected after superovulation whereas few embryos were obtained post mating [28].

Results shown in Fig. 5 are for eggs after removal of the zona pellucida. It can be seen in Fig. 5B that wild type ovulated eggs bound substantially more L-PHA than mature blastocysts which served as the negative control for SSEA-3 MAb binding (Fig. 5B and 5C, dashed arrows). SSEA-3 was well expressed on wild type eggs (Fig. 5C and 5C'). Mgat1 mutant eggs bound L-PHA poorly (Fig. 5E and 5E') but bound the SSEA-3 MAb similarly to wild type eggs (Fig. 5F and 5F'). The control blastocysts did not bind the SSEA-3 MAb (Fig. 5C, 5F, 5C ' and $5 \mathrm{~F}^{\prime}$, dashed arrows). In a separate experiment in which all 4 early embryos obtained from a gatl $^{\mathrm{F} / \mathrm{F}}: \mathrm{ZP} 3 \mathrm{Cre}$ female mated to an $\mathrm{Mgat}^{+/-}$male were tested, each embryo bound the SSEA-3 MAb similarly to control embryos (not shown). Therefore SSEA-3 was expressed well in eggs and early embryos lacking complex N-glycans and they are not the major carriers of the SSEA-3 epitope during oogenesis or embryogenesis.

\section{Expression of SSEA-4 in mouse embryos lacking core 1-derived 0-glycans}

SSEA-4 is expressed on eggs and preimplantation embryos until the blastocyst stage in a pattern similar to SSEA-3 [21,24]. To determine if the SSEA-4 epitope is carried on core 1-derived O-glycans, embryos lacking these glycans were generated by crossing females ovulating $T$ syn $^{-/-}$eggs with $T$-syn ${ }^{+-}$males. $T$-syn mutant embryos at the 4-8 cell stage were identified by their low PNA binding (circled in Fig. 6E and 6F). They bound the SSEA-4 MAb well (Fig. $6 \mathrm{~F}$ and $6 \mathrm{~F}^{\prime}$, solid arrows). Mature negative control blastocysts bound only low levels of SSEA-4 MAb (Fig. 6C, 6C', 6F and 6F', dashed arrows). Control embryos and $T$-syn mutant embryos were similar in their SSEA-4 MAb binding, as were control and mutant eggs (Fig. 6C, 6C', 6F and $\left.6 \mathrm{~F}^{\prime}\right)$. Therefore, core 1-derived $\mathrm{O}$-glycans are not essential for the expression of the SSEA-4 epitope during oogenesis or on early mouse embryos. 


\section{Expression of SSEA-4 in mouse eggs and embryos lacking complex and hybrid $\mathrm{N}$-glycans}

Eggs collected from Mgatl mutant females lack complex and hybrid N-glycans and did not bind L-PHA (Fig. 7E). Control eggs bound L-PHA as expected (Fig. 7B), and exhibited much higher fluorescence than the negative control blastocyst in Fig. 7E. The control blastocyst bound very low amounts of SSEA-4 MAb (Fig. 7F, dashed arrow). By contrast both Mgat $^{-1-}$ mutant and wild type eggs bound the SSEA-4 MAb well and exhibited equivalent

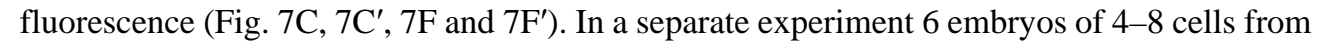
a cross between a $\mathrm{Mgatl}^{\mathrm{F} / \mathrm{F}}: \mathrm{ZP} 3 \mathrm{Cre}$ female and a $\mathrm{Mgatl}^{+/-}$male were all SSEA-4 strongly positive (not shown). Therefore, complex $\mathrm{N}$-glycans are not essential for the expression of the SSEA-4 epitope on ovulated eggs or early preimplantation mouse embryos.

\section{Expression of $\mathrm{Le}^{\mathrm{Y}}$ in mouse embryos lacking core 1-derived O-glycans}

The $\mathrm{Le}^{\mathrm{Y}}$ antigen is expressed on blastocysts prior to implantation $[18,27]$. Therefore blastocysts generated from control and T-syn mutant females were compared for binding of a $\mathrm{MAb}$ against $\mathrm{Le}^{\mathrm{Y}}$. Earlier stage embryos that do not express $\mathrm{Le}^{\mathrm{Y}}$ were included as negative controls (Fig. 8C, 8C' and 8F, dashed arrows). Control blastocysts bound the $\mathrm{Le}^{\mathrm{Y}} \mathrm{MAb}$ well (Fig. $8 \mathrm{C}$ and $8 \mathrm{C}^{\prime}$ ) and so did $T$-syn mutant blastocysts (Fig. $8 \mathrm{~F}$ and $8 \mathrm{~F}^{\prime}$, solid arrows) identified by their lack of PNA binding (circled in Fig. 8E, and 8E'). Similar results were found for 17 mutant blastocysts. Therefore, glycoproteins do not carry the $\mathrm{Le}^{\mathrm{Y}}$ epitope solely on their core 1-derived O-glycans.

\section{Expression of $\mathrm{Le}^{\mathrm{Y}}$ in mouse embryos lacking complex and hybrid $\mathrm{N}$-glycans}

$\mathrm{Le}^{\mathrm{Y}}$ was also not carried solely on complex N-glycans. Early embryos that did not express $\mathrm{Le}^{\mathrm{Y}}$ did not bind the $\mathrm{Le}^{\mathrm{Y}} \mathrm{MAb}$ (Fig. 9C, 9 $\mathrm{C}^{\prime}, 9 \mathrm{~F}$ and $9 \mathrm{~F}^{\prime}$, dashed arrows). Blastocysts collected from Mgatl mutant females and lacking L-PHA binding (circled in Fig. 9E and 9F) bound the $\mathrm{Le}^{\mathrm{Y}} \mathrm{MAb}$ as well as control blastocysts (Fig. $9 \mathrm{C}$ and $9 \mathrm{C}^{\prime}$ compared to $9 \mathrm{~F}$ and $9 \mathrm{~F}^{\prime}$, solid arrows). As previously observed, $\sim 50 \%$ of the blastocysts generated by $\mathrm{Mgat}^{\mathrm{F} / \mathrm{F}}$ :ZP3 Cre females (Fig. 9D) had irregular morphology [28], however this did not alter the stage-specific expression of $\mathrm{Le}^{\mathrm{Y}}$ (Fig. 9F). Therefore the $\mathrm{Le}^{\mathrm{Y}}$ epitope is not predominantly expressed on the $\mathrm{N}$-glycans or O-glycans (shown above) of glycoproteins.

\section{Discussion}

Determining molecular parameters of preimplantation mouse embryos is a challenge due to the difficulties of obtaining sufficient numbers of staged embryos. Structural analyses of glycans of the zona pellucida have been performed but required very large numbers of mice [35]. Thus most evidence concerning structural features of mammalian developmental antigens have been obtained by indirect approaches. In this work we have examined the expression of developmentally-regulated SSEAs of the mouse embryo by characterizing the expression of glycan antigens recognized by monoclonal antibodies in maternal and zygotic mutant embryos that are missing a key enzyme necessary for the synthesis of entire classes of $\mathrm{N}$ - or O-glycans. The female mice used to generate mutant embryos have been phenotypically characterized in some depth previously [28,29,32] (Williams and Stanley, submitted). Mutant embryos were identified by their inability to bind lectins that recognize complex N-glycans or core $1 \mathrm{O}-$ glycans and characterized for MAb binding by indirect immunofluorescence. MAb specificity was demonstrated from background binding to embryos at particular stages of development known from previous work not to express the relevant antigen (reviewed in $[7,24]$ ). Therefore, the dynamic range of the experiments was quite large.

Interestingly, despite the fact that there is much evidence that each SSEA examined is expressed on glycoproteins in cells and tissues, we observed no marked reduction in MAb binding in mutant eggs or embryos lacking complex N- or core 1-derived O-glycans. Thus when T- 
synthase was absent and eggs or embryos did not express core 1-derived O-glycans, 8-cell compacted embryos bound SSEA-1 MAb, eggs and early embryos bound SSEA-3 and SSEA-4 $\mathrm{MAbs}$, and mature blastocysts bound the $\mathrm{Le}^{\mathrm{Y}} \mathrm{MAb}$ equivalently to control embryos at the same stage. Therefore, core 1-derived O-glycans on glycoproteins are not essential for the expression of these SSEAs. A similar conclusion was reached with eggs and staged embryos lacking complex and hybrid $\mathrm{N}$-glycans. It would have been very helpful to have examined embryos lacking both classes of glycan together. Unfortunately, however, the fertility of females conditionally mutant for both T-syn and Mgat1 is extremely low [29], and it was not possible to obtain staged embryos from these females for these experiments. Nevertheless, the fact that mutant and control MAb binding was equivalent for each SSEA in both of the single conditional mutants suggests that SSEA-1, SSEA-3, SSEA-4 and Le ${ }^{\mathrm{Y}}$ are predominantly expressed on glycolipids in preimplantation mouse embryos. This question can be examined directly by crossing female mice conditionally mutant for glucosylceramide synthase [36] with ZP3Cre recombinase transgenic mice and determining SSEA expression on null embryos at different stages of development.

While the data presented in this work predict that potentially no glycoproteins carry the SSEA glycan epitopes examined, previous experiments have reported that several glycoproteins from mouse embryos labeled by ${ }^{125} \mathrm{I}$ and lactoperoxidase are immunoprecipitated by the SSEA-3 MAb [23]. Evidence that N-glycans may carry the SSEA-3 epitope comes from the fact that treatment with tunicamycin reduced the expression of SSEA-3 on fucose-labeled glycoproteins from an embryonal carcinoma line [23]. While similar experiments have not been reported for SSEA-1, SSEA-4 or Le ${ }^{\mathrm{Y}}$, it is known that at least the SSEA-1 [37] and Le ${ }^{\mathrm{Y}}$ epitopes are expressed on $\mathrm{N}$-glycans of numerous glycoproteins. Both SSEA-3 and SSEA-4 epitopes are commonly found on globo-series glycolipids [21] and are predicted to be expressed on glycolipids of the mouse embryo [22]. The data we present here are consistent with this prediction. Thus, in summary, maternal and zygotic embryos that do not express complex or hybrid N-glycans or core 1-derived O-glycans, express the glycan epitopes recognized by the SSEA-1, SSEA-3, SSEA-4 and Le ${ }^{Y}$ MAbs at levels equivalent to control embryos. Therefore neither class of glycan is essential for the expression of these SSEAs by cells of the mouse embryo.

\section{Acknowledgments}

We thank Wen Dong for excellent technical assistance and Dr. Jason Aglipay for help with some experiments. This work was supported by grant RO1 30645 from the National Institutes of Health to P. S. and received partial support from the Albert Einstein Cancer Center grant PO1 13330.

\section{Abbreviations}

$\begin{array}{ll}\text { SSEA } & \text { stage-specific embryonic antigen } \\ \mathrm{Le}^{\mathrm{X}} & \text { Lewis } \mathrm{X} \\ \mathrm{Le}^{\mathrm{Y}} & \text { Lewis Y } \\ \text { L-PHA } & \text { leukoagglutinin from P. vulgaris } \\ \text { PNA } & \text { peanut agglutinin } \\ \text { MAb } & \text { monoclonal antibody } \\ \text { FITC } & \text { fluoroesceinisothiocyanate } \\ \text { TRITC } & \text { tetramethylrhodamine isothiocyanate }\end{array}$




\section{References}

1. Solter D, Knowles BB. Monoclonal antibody defining a stage-specific mouse embryonic antigen (SSEA-1). Proc Natl Acad Sci USA 1978;75:5565-5569. [PubMed: 281705]

2. Solter D, Knowles BB. Developmental stage-specific antigens during mouse embryogenesis. Curr Top Dev Biol 1979;13:139-165. [PubMed: 396115]

3. Pennington JE, Rastan S, Roelcke D, Feizi T. Saccharide structures of the mouse embryo during the first eight days of development. Inferences from immunocytochemical studies using monoclonal antibodies in conjunction with glycosidases. J Embryol \& Exp Morph 1985;90:335-361. [PubMed: 3009679]

4. Muramatsu T. Developmentally regulated expression of cell surface carbohydrates during mouse embryogenesis. J Cellular Biochem 1988;36:1-14. [PubMed: 3277983]

5. Muramatsu T, Muramatsu H. Carbohydrate antigens expressed on stem cells and early embryonic cells. Glycoconj J 2004;21:41-45. [PubMed: 15467397]

6. Fenderson BA, O'Brien DA, Millette CF, Eddy EM. Stage-specific expression of three cell surface carbohydrate antigens during murine spermatogenesis detected with monoclonal antibodies. Developmental Biology 1984;103:117-128. [PubMed: 6370756]

7. Brown DG, Warren VN, Pahlsson P, Kimber SJ. Carbohydrate antigen expression in murine embryonic stem cells and embryos. I. Lacto and neo-lacto determinants. Histochemical J 1993;25:452-463.

8. Gooi HC, Feizi T, Kapadia A, Knowles BB, Solter D, Evans MJ. Stage-specific embryonic antigen involves alpha 1 goes to 3 fucosylated type 2 blood group chains. Nature 1981;292:156-158. [PubMed: 6165896]

9. Hakomori S, Nudelman E, Levery S, Solter D, Knowles BB. The hapten structure of a developmentally regulated glycolipid antigen (SSEA-1) isolated from human erythrocytes and adenocarcinoma: a preliminary note. Biochem Biophys Res Commun 1981;100:1578-1586. [PubMed: 6117281]

10. Fenderson BA, Zehavi U, Hakomori S. A multivalent lacto-N-fucopentaose III-lysyllysine conjugate decompacts preimplantation mouse embryos, while the free oligosaccharide is ineffective. Journal of Experimental Medicine 1984;160:1591-1596. [PubMed: 6491606]

11. Bird JM, Kimber SJ. Oligosaccharides containing fucose linked alpha(1-3) and alpha(1-4) to Nacetylglucosamine cause decompaction of mouse morulae. Dev Biol 1984;104:449-460. [PubMed: 6745495]

12. Kudo T, Kaneko M, Iwasaki H, Togayachi A, Nishihara S, Abe K, Narimatsu H. Normal embryonic and germ cell development in mice lacking alpha 1,3-fucosyltransferase IX (Fut9) which show disappearance of stage-specific embryonic antigen 1. Mol Cell Biol 2004;24:4221-4228. [PubMed: 15121843]

13. Feizi T. The antigens Ii, SSEA-1 and ABH are in interrelated system of carbohydrate differentiation antigens expressed on glycosphingolipids and glycoproteins. Adv Exp Med Biol 1982;152:167-177. [PubMed: 6127917]

14. Andrews, PW.; Knowles, BB.; Cossu, G.; Solter, D. Teratocarcinoma and mouse embryo cell surface antigens: Characterization of the molecule(s) carrying the SSEA-1 antigenic determinant. In: Muramatsu, T.; Gachelin, G.; Mosocima, AA.; Ikawa, Y., editors. Teratocarcinoma and Embryonic Cell Interactions. Japan Scientific Societies Press; Tokyo: 1982. p. 103-119.

15. Childs RA, Pennington J, Uemura K, Goddfellow PN, Evans MJ, Feizi T. High Molecular-weight glycoproteins are the major carriers of the carbohydrate differentiation antigens I, i, and SSEA-1 of mouse teratocarcinoma cells. Biochem J 1983;215:491-503. [PubMed: 6197967]

16. Feizi T. Demonstration by monoclonal antibodies that carbohydrate structures of glycoproteins and glycolipids are onco-developmental antigens. Nature 1985;314:53-57. [PubMed: 2579340]

17. Ozawa M, Muramatsu T, Solter D. SSEA-1, a stage-specific embryonic antigen of the mouse, is carried by the glycoprotein-bound large carbohydrate in embryonal carcinoma cells. Cell Differ 1985;16:169-173. [PubMed: 2860973]

18. Kimber SJ, MacQueen HA, Bagley PR. Fucosylated glycoconjugates in mouse preimplantation embryos. J Exp Zool 1987;244:395-408. [PubMed: 3443830] 
19. Fenderson BA, Eddy EM, Hakomori S. The blood group I antigen defined by monoclonal antibody C6 is a marker of early mesoderm during murine embryogenesis. Differentiation 1988;38:124-133. [PubMed: 3209003]

20. Fenderson BA, Eddy EM, Hakomori S. Glycoconjugate expression during embryogenesis and its biological significance. Bioessays 1990;12:173-179. [PubMed: 1970725]

21. Kannagi R, Cochran NA, Ishigami F, Hakomori S, Andrews PW, Knowles BB, Solter D. Stagespecific embryonic antigens (SSEA-3 and -4) are epitopes of a unique globo-series ganglioside isolated from human teratocarcinoma cells. EMBO J 1983;2:2355-2361. [PubMed: 6141938]

22. Kannagi R, Levery SB, Ishigami F, Hakomori S, Shevinsky LH, Knowles BB, Solter D. New globoseries glycosphingolipids in human teratocarcinoma reactive with the monoclonal antibody directed to a developmentally regulated antigen, stage-specific embryonic antigen 3. J Biol Chem 1983;258:8934-8942. [PubMed: 6863318]

23. Shevinsky LH, Knowles BB, Damjanov I, Solter D. Monoclonal antibody to murine embryos defines a stage-specific embryonic antigen expressed on mouse embryos and human teratocarcinoma cells. Cell 1982;30:697-705. [PubMed: 6183004]

24. Kimber SJ, Brown DG, Pahlsson P, Nilsson B. Carbohydrate antigen expression in murine embryonic stem cells and embryos. II. Sialylated antigens and glycolipid analysis. Histochemical J 1993;25:628641.

25. Abe K, McKibbin JM, Hakomori S. The monoclonal antibody directed to difucosylated type 2 chain (Fuc alpha 1 leads to 2Gal beta 1 leads to 4[Fuc alpha 1 leads to 3]GlcNAc; Y Determinant). J Biol Chem 1983;258:11793-11797. [PubMed: 6194156]

26. Wang XQ, Zhu ZM, Fenderson BA, Zeng GQ, Cao YJ, Jiang GT. Effects of monoclonal antibody directed to LeY on implantation in the mouse. Mol Hum Reprod 1998;4:295-300. [PubMed: 9570276]

27. Zhu ZM, Kojima N, Stroud MR, Hakomori SI, Fenderson BA. Monoclonal antibody directed to LeY oligosaccharide inhibits implantation in the mouse. Biol Reprod 1995;52:903-912. [PubMed: 7780013]

28. Shi S, Williams SA, Seppo A, Kurniawan H, Chen W, Ye Z, Marth JD, Stanley P. Inactivation of the Mgat1 gene in oocytes impairs oogenesis, but embryos lacking complex and hybrid N-glycans develop and implant. Mol Cell Biol 2004;24:9920-9929. [PubMed: 15509794]

29. Williams SA, Xia L, Cummings RD, McEver RP, Stanley P. Fertilization in mouse does not require terminal galactose or N-acetylglucosamine on the zona pellucida glycans. J Cell Sci 2007;120:13411349. [PubMed: 17374637]

30. Philpott CC, Ringuette MJ, Dean J. Oocyte-specific expression and developmental regulation of ZP3, the sperm receptor of the mouse zona pellucida. Dev Biol 1987;121:568-575. [PubMed: 2884155]

31. de Vries WN, Binns LT, Fancher KS, Dean J, Moore R, Kemler R, Knowles BB. Expression of Cre recombinase in mouse oocytes: a means to study maternal effect genes. Genesis 2000;26:110-112. [PubMed: 10686600]

32. Williams SA, Stanley P. Mouse fertility is enhanced by oocyte-specific loss of core 1-derived Oglycans. Faseb J 2008;22:2273-2284. [PubMed: 18276833]

33. De Vries WN, Evsikov AV, Haac BE, Fancher KS, Holbrook AE, Kemler R, Solter D, Knowles BB. Maternal beta-catenin and E-cadherin in mouse development. Development 2004;131:4435-4445. [PubMed: 15306566]

34. Ioffe E, Liu Y, Stanley P. Complex N-glycans in Mgat1 null preimplantation embryos arise from maternal Mgat1 RNA. Glycobiology 1997;7:913-919. [PubMed: 9363433]

35. Dell A, Chalabi S, Easton RL, Haslam SM, Sutton-Smith M, Patankar MS, Lattanzio F, Panico M, Morris HR, Clark GF. Murine and human zona pellucida 3 derived from mouse eggs express identical O-glycans. Proc Natl Acad Sci U S A 2003;100:15631-15636. [PubMed: 14673092]

36. Yamashita T, Allende ML, Kalkofen DN, Werth N, Sandhoff K, Proia RL. Conditional LoxP-flanked glucosylceramide synthase allele controlling glycosphingolipid synthesis. Genesis 2005;43:175180. [PubMed: 16283624]

37. Fenderson BA, Radin N, Andrews PW. Differentiation antigens of human germ cell tumours: distribution of carbohydrate epitopes on glycolipids and glycoproteins analyzed using PDMP, an inhibitor of glycolipid synthesis. Eur Urol 1993;23:30-36. [PubMed: 7682952] 

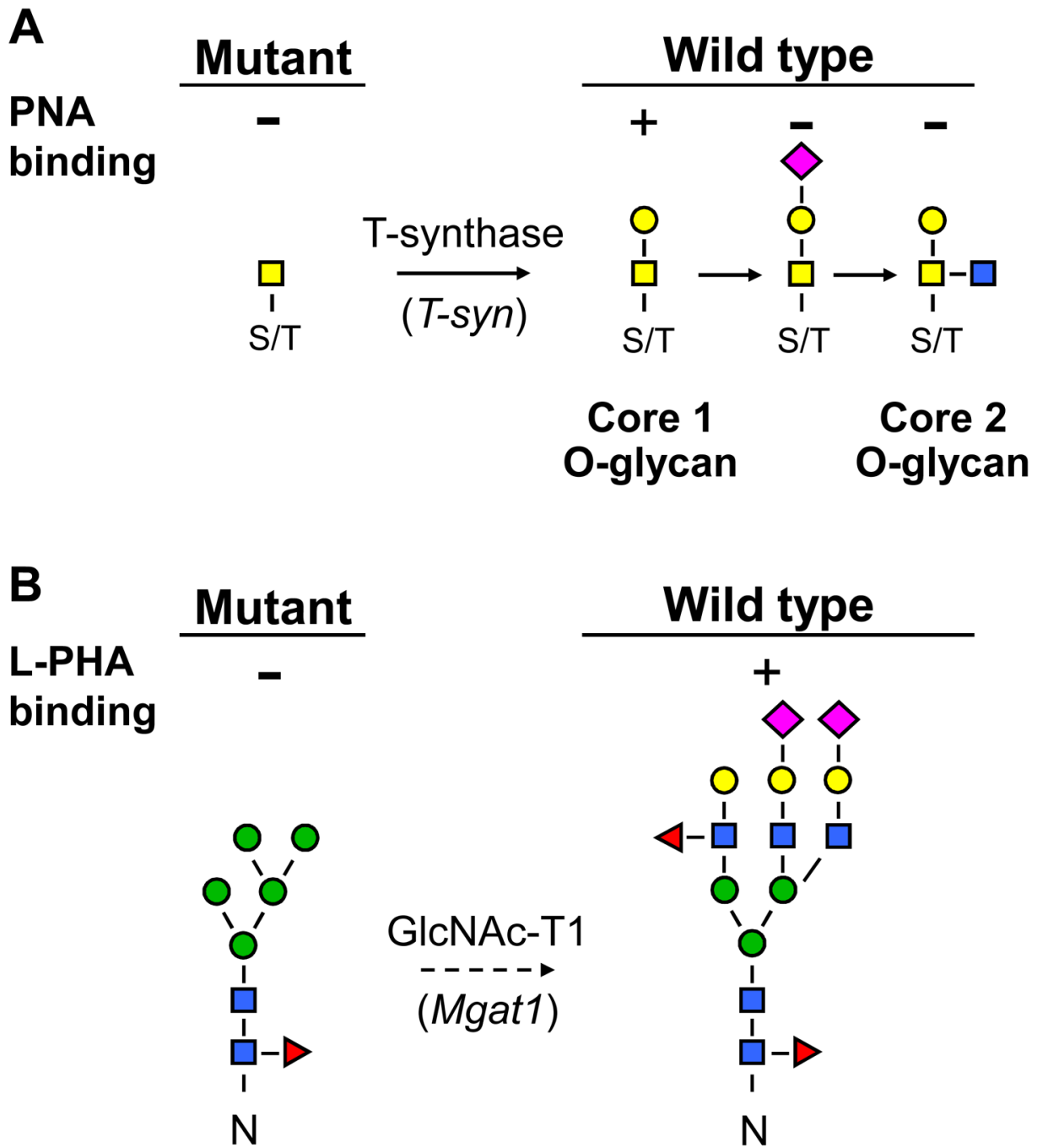

\section{Oligomannose $\mathrm{N}$-glycan}

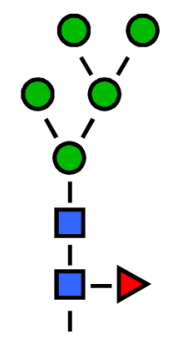

$\mathrm{N}$

$\mathrm{N}$-acetylglucosamine

$\square \mathrm{N}$-acetylgalactosamine
OMannose

OGalactose
Complex

$\mathrm{N}$-glycan

Fig. 1.

Detection of glycans expressed by wild type and mutant embryos. A. T-synthase (T-syn) initiates the synthesis of core 1-derived O-glycans. The core $1 \mathrm{O}$-glycan binds PNA. Wild type eggs bind PNA but eggs lacking T-syn do not [29]. If sialic acid is added to form core 1 or GlcNAc to form core 2, PNA binding is inhibited. B. Mgatl initiates the synthesis of complex and hybrid N-glycans. The dotted arrow signifies that more than one biochemical step is involved. Complex N-glycans bind L-PHA. Wild type eggs and embryos bind L-PHA but mutants lacking Mgatl do not [28,29,34]. 


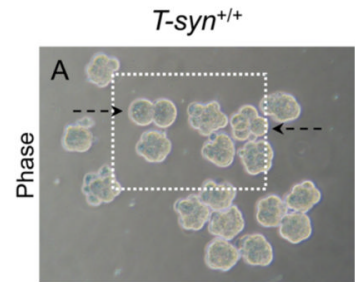

T-syn+/- or T-syn ${ }^{-/}$
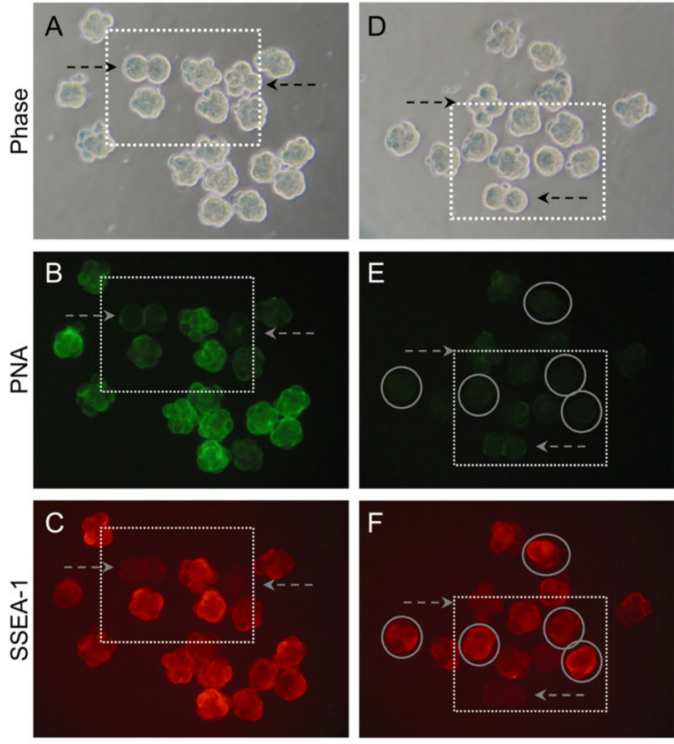
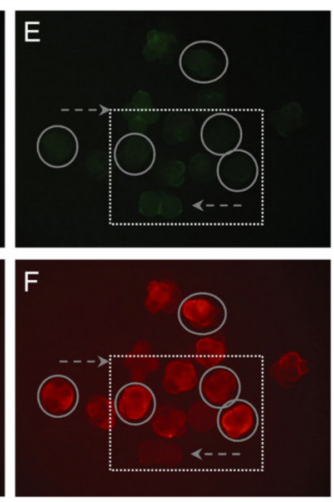

T-syn+/+
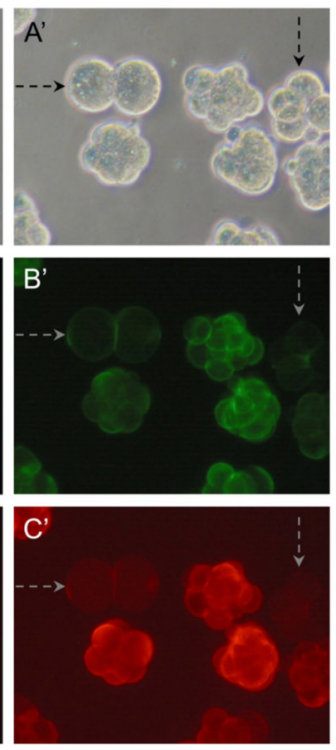

T-syn ${ }^{+/-}$or $T-$ syn $^{-1-}$
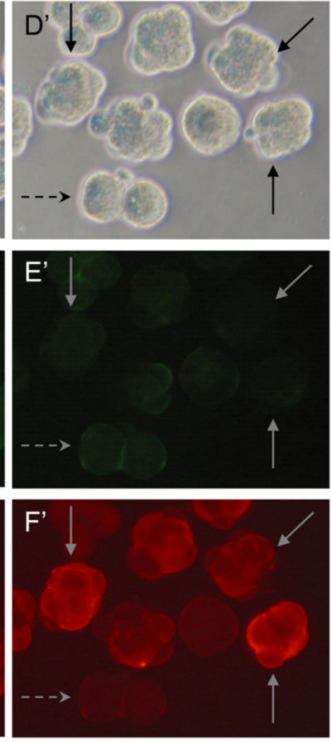

Fig. 2.

SSEA-1 expression on 8-cell compacted control and $T$-syn ${ }^{-1-}$ embryos. SSEA-1-negative controls were 2- and 4-cell wild type embryos that were included with both control and mutant embryo samples (dashed arrows). A. Phase contrast of 8-cell compacted control embryos obtained from $T-s y n^{\mathrm{F} / \mathrm{F}}$ females mated to $T-s y n^{+/-}$males. B. Control embryos binding PNA. C. Control embryos binding the SSEA-1 MAb. Only the compacted embryos fluoresced brightly. D Phase contrast of 8-cell compacted embryos obtained from T-syn ${ }^{\mathrm{F} / \mathrm{F}}: \mathrm{ZP} 3 C r e$ mutant females mated with $T-s y n^{+/-}$males. E. The same $T-s y n^{+/-}$and $T-s y n^{-1-}$ embryos binding PNA. PNA-negative $T$-syn ${ }^{-/-}$compacted 8-cell embryos are circled. F. The same $T$-syn ${ }^{+/}$and $T$ $s y n^{-l-}$ embryos binding the SSEA-1 MAb. $\mathrm{A}^{\prime}-\mathrm{F}^{\prime}$. The area marked by each dotted square in $\mathrm{A}-\mathrm{F}$ is magnified. Images are at the same magnification and the same exposure. The embryos shown were from one of two experiments that compared 24 control compacted 8-cell embryos from 5 females and $20 \mathrm{~T}$-syn mutants and heterozygous compacted embryos from 4 mutant females. 

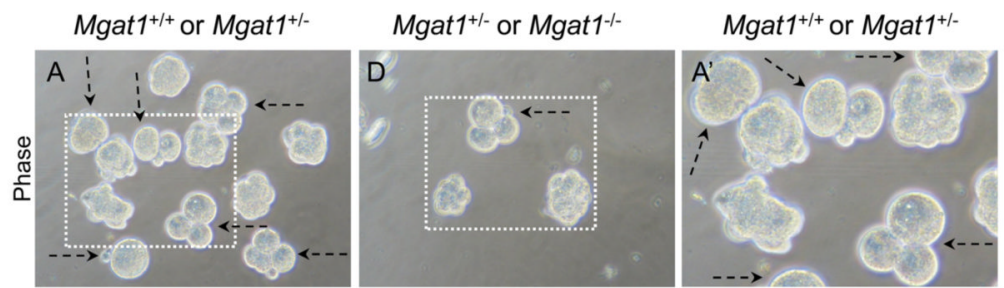

Mgat ${ }^{+/-}$or Mgat $1^{1--}$
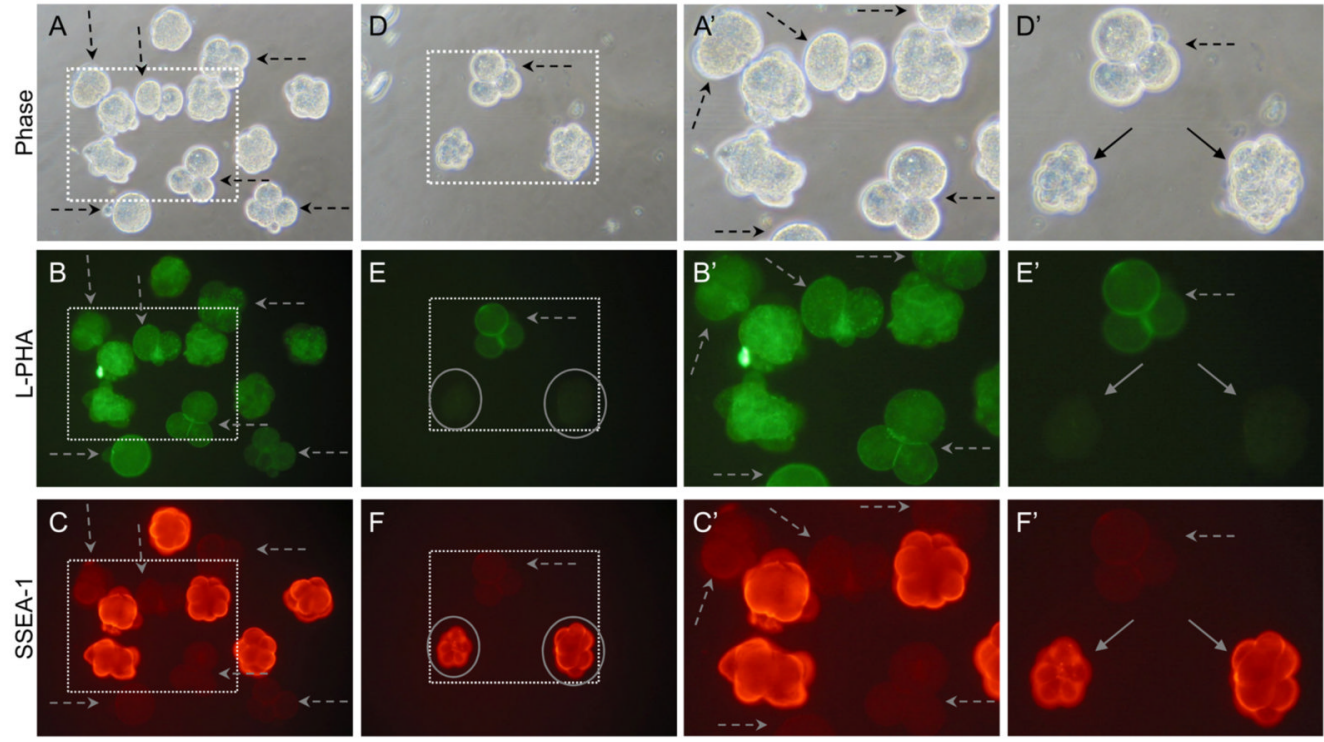

Fig. 3.

SSEA-1 expression on 8-cell compacted embryos from Mgat1 mutant females. SSEA-1negative 2-4 cell embryos were included with both control and mutant embryos (dashed arrows). A. Phase contrast of 8-cell compacted control embryos obtained from Mgatt $^{\mathrm{F} / \mathrm{F}}$ females mated with $\mathrm{MgatI}^{+-}$males. B. The wild type or heterozygous embryos from A with L-PHA. C. The embryos from A with the SSEA-1 MAb. D. Phase contrast of 8-cell compacted embryos obtained from $\mathrm{MgatI}^{\mathrm{F} / \mathrm{F}}: \mathrm{ZP} 3 \mathrm{Cr}$ e females mated with $\mathrm{MgatI}^{+/-}$males. E. The embryos from D binding L-PHA. Both 8-cell compacted embryos were L-PHA negative (circled). F. The embryos from D binding the SSEA-1 MAb. L-PHA-negative embryos (circled) fluoresce brightly. $A^{\prime}-F^{\prime}$. The area marked by each dotted square in A-F is magnified. Images are at the same magnification and the same exposure. The embryos shown were from one of two experiments that compared 12 control compacted 8-cell embryos from 4 females and 8 Mgat 1 mutant and heterozygous compacted embryos from 3 mutant females. 

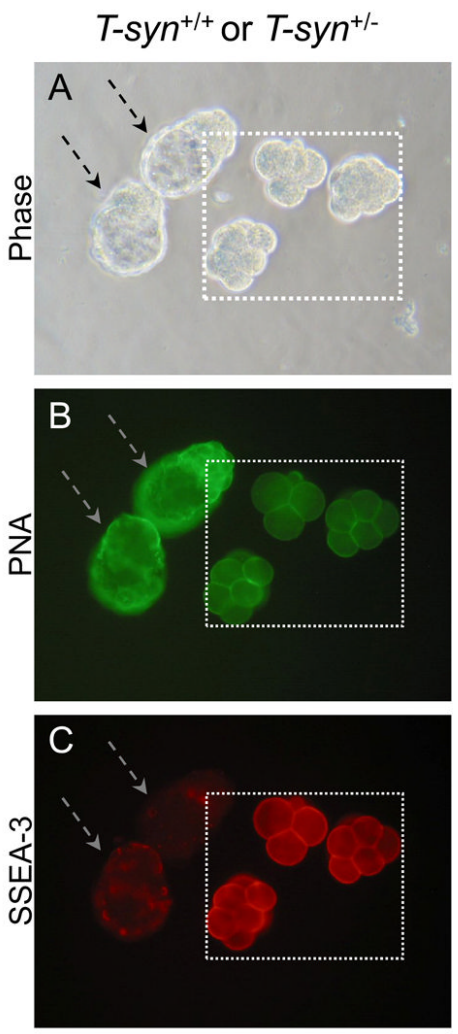

T-syn ${ }^{+/-}$or T-syn ${ }^{-/}$

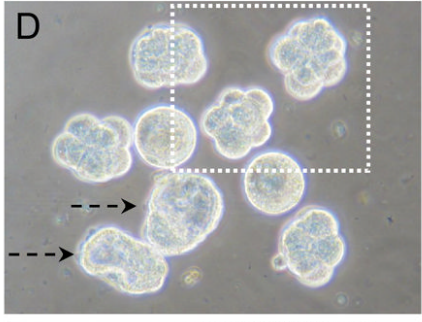

T-syn ${ }^{+/+}$or $\mathrm{T}-\mathrm{syn}^{+/-}$
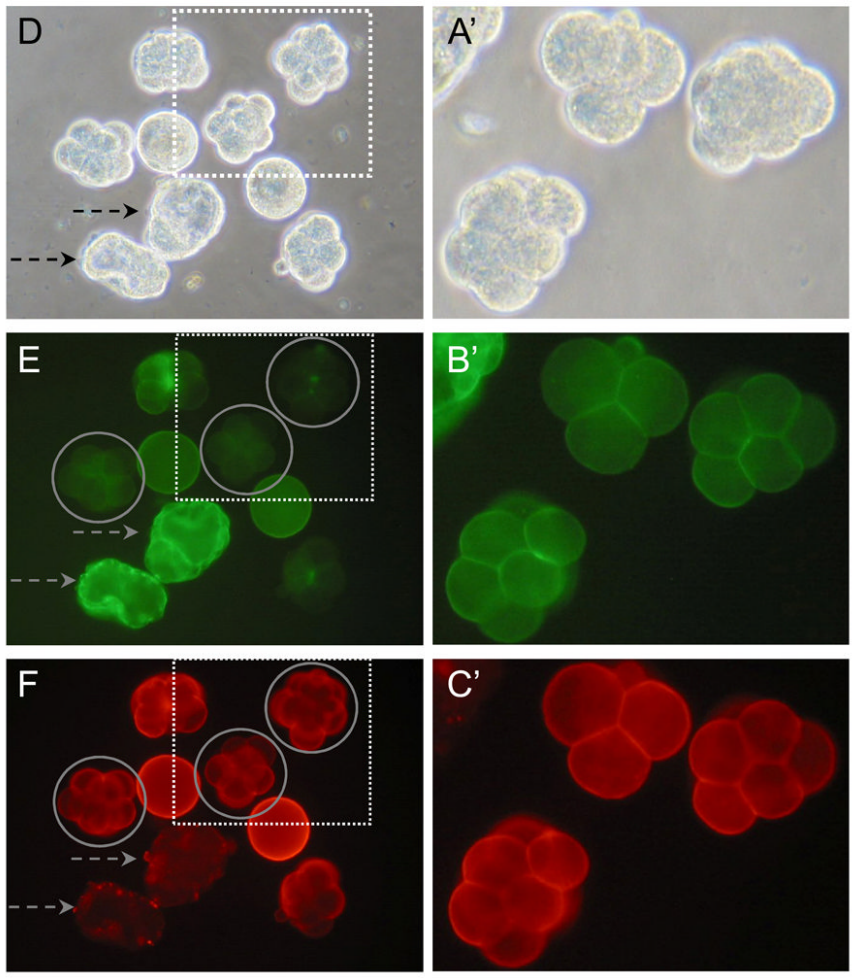
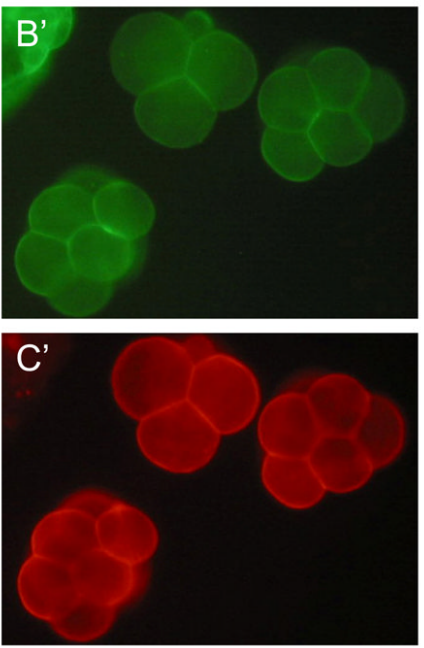

T-syn+/- or T-syn ${ }^{-/-}$
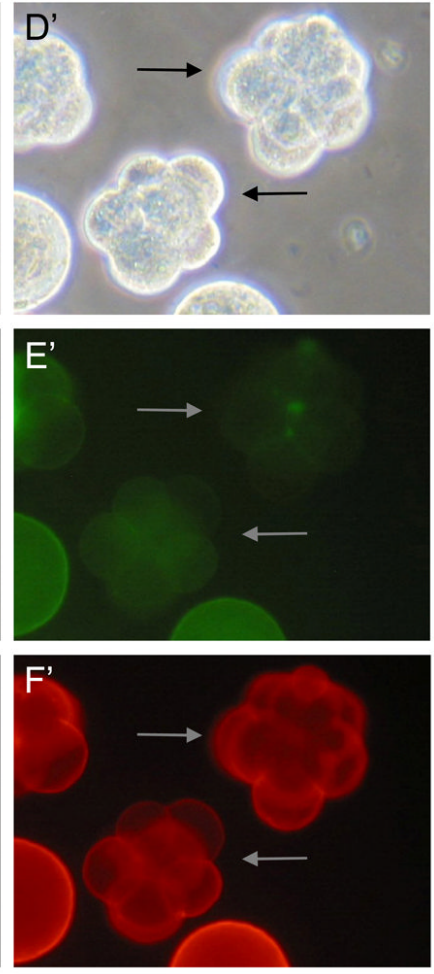

Fig. 4.

SSEA-3 expression on embryos from control and T-syn mutant females. Late-stage blastocysts were included as negative controls (dashed arrows). A. Phase contrast of control embryos obtained from $T$-syn ${ }^{\mathrm{F} / \mathrm{F}}$ females mated to $T$-syn ${ }^{+/-}$males. B. Control embryos from A binding PNA. C. Control embryos from A binding the SSEA-3 MAb. Only the 4-8 cell embryos fluoresced brightly. D. Phase contrast of embryos obtained from $T$-syn ${ }^{\mathrm{F} / \mathrm{F}}: \mathrm{ZP} 3 \mathrm{Cre}$ mutant females mated with $T-s y n^{+-}$males. E. The $T-s y n^{+/-}$and $T-s y n^{-1-}$ embryos from D binding PNA. PNA-negative $T$-syn ${ }^{-1-}$ embryos are circled. F. The same $T-$ syn $^{+/-}$and $T-s y n^{-1-}$ embryos from D binding the SSEA-3 MAb. PNA-negative SSEA-3-positive $T$-syn ${ }^{-1-}$ embryos are circled. $A^{\prime}-F^{\prime}$. The area marked by each dotted square in A-F is magnified. Images are at the same magnification and the same exposure. Solid arrows indicate PNA-negative mutant embryos. The images are representative of 3 independent experiments with 2 eggs and 214 8 cell embryos, half of the total generated by 6 control females, 3 eggs and $214-8$ cell embryos, half of the total generated by $6 T$-syn mutant females. 

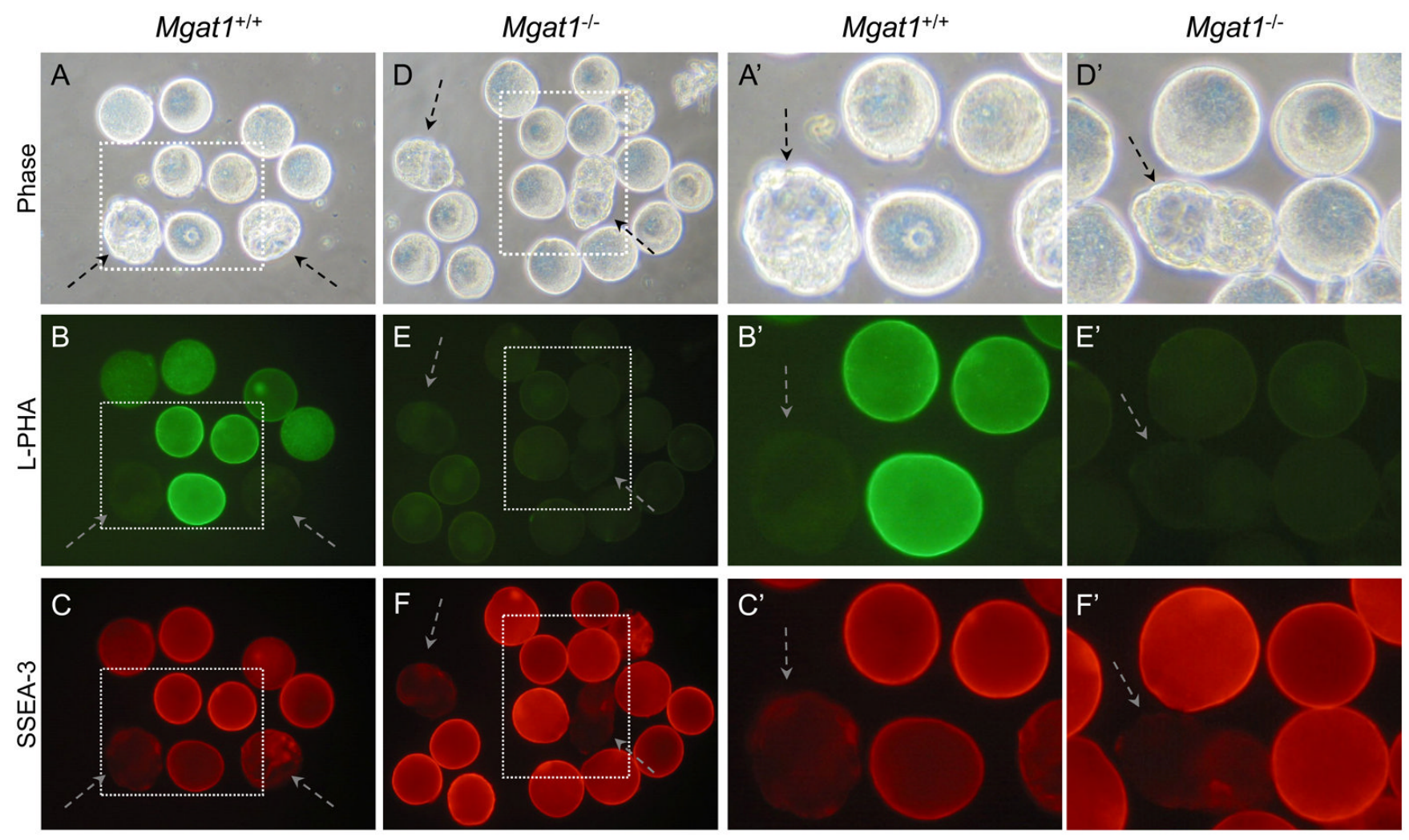

Fig. 5.

SSEA-3 expression on ovulated eggs from $M$ gat 1 mutant females. Mgat $^{\mathrm{F} / \mathrm{F}}: \mathrm{ZP} 3 \mathrm{Cre}$ females were superovulated and eggs collected. Wild type blastocysts (dashed arrows) were included as negative controls for SSEA-3 MAb binding. A. Phase contrast of control zona-free eggs obtained from superovulated $M g a t 1^{\mathrm{F} / \mathrm{F}}$ females. B. L-PHA binding to control eggs from A. C. SSEA-3 MAb binding to control eggs from A. D. Phase contrast of zona-free eggs obtained from Mgat $^{\mathrm{F} / \mathrm{F}}$ :ZP3Cre females. E. L-PHA binding to the mutant eggs from D. F. SSEA-3 MAb binding to the mutant eggs from D. L-PHA negative eggs fluoresce strongly. $\mathrm{A}^{\prime}-\mathrm{F}^{\prime}$. The area marked by each dotted square in A-F is magnified. Images are at the same magnification and the same exposure and are rotated when necessary. Images are representative of 3 experiments with 23 eggs, a 6-cell and a compacted 8-cell embryo, half of the total generated by 11 control females, and 17 eggs and 4, 4-6 cell embryos, half of the total generated by 8 mutant females. 


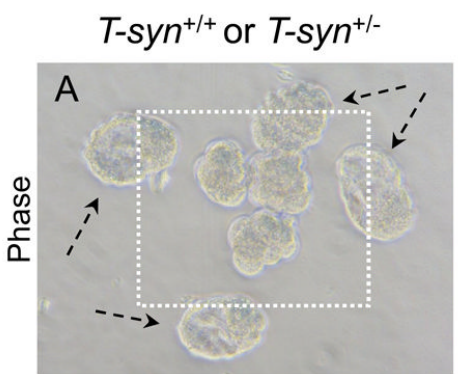

$T-s y n^{+/-}$or $T-s y n^{-1-}$
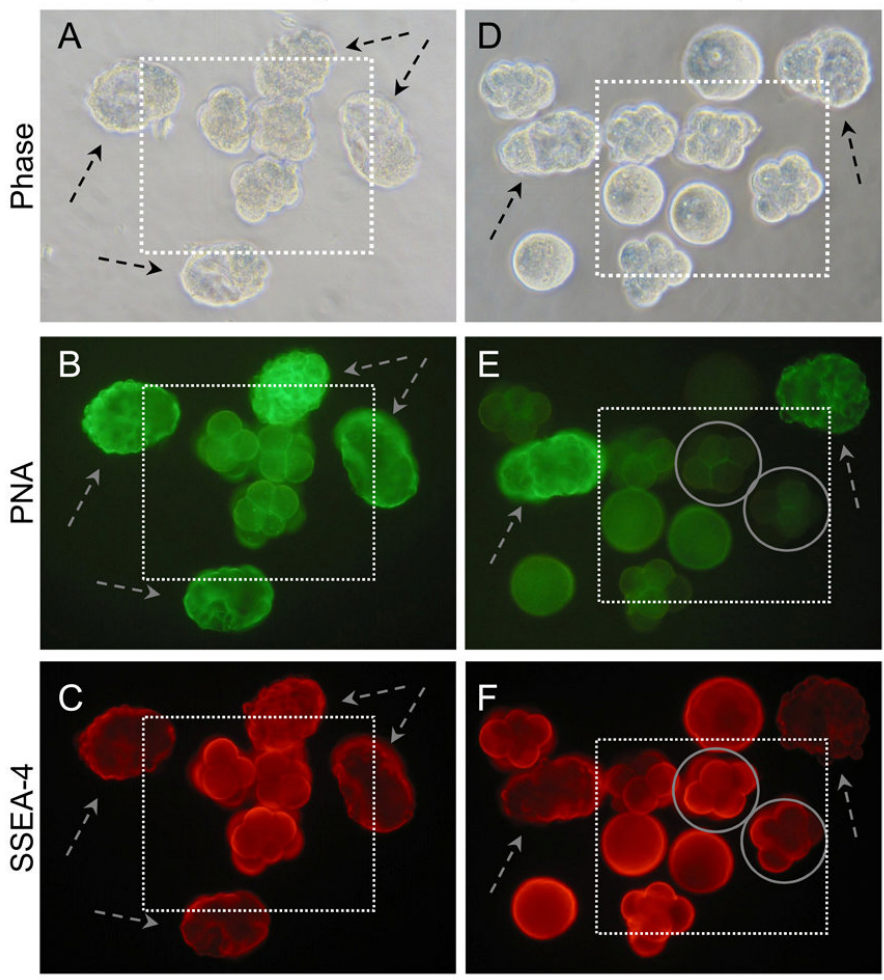
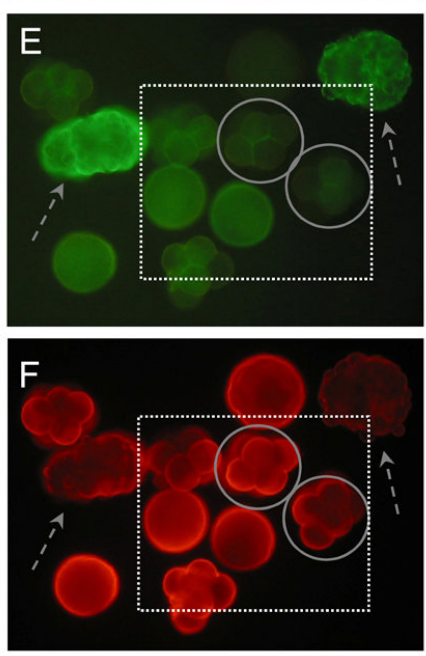

T-syn ${ }^{+/+}$or $T$-syn ${ }^{+/-}$
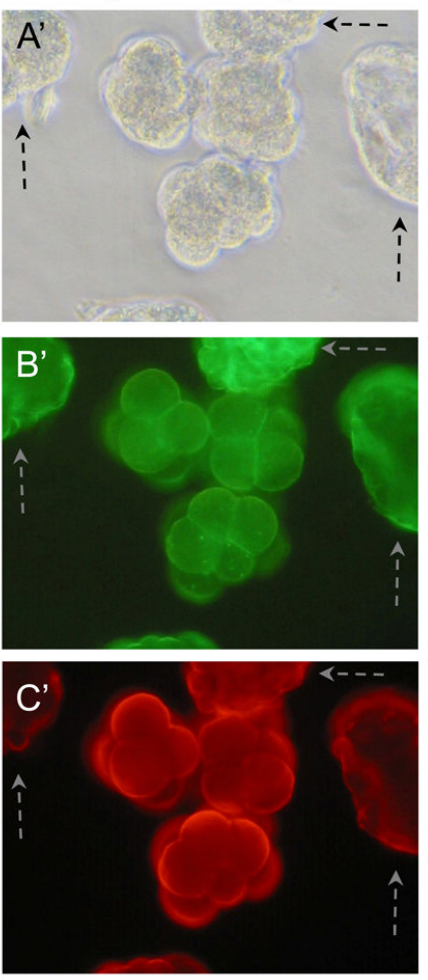

$T-s y n^{+/-}$or $T-s y n^{-1-}$
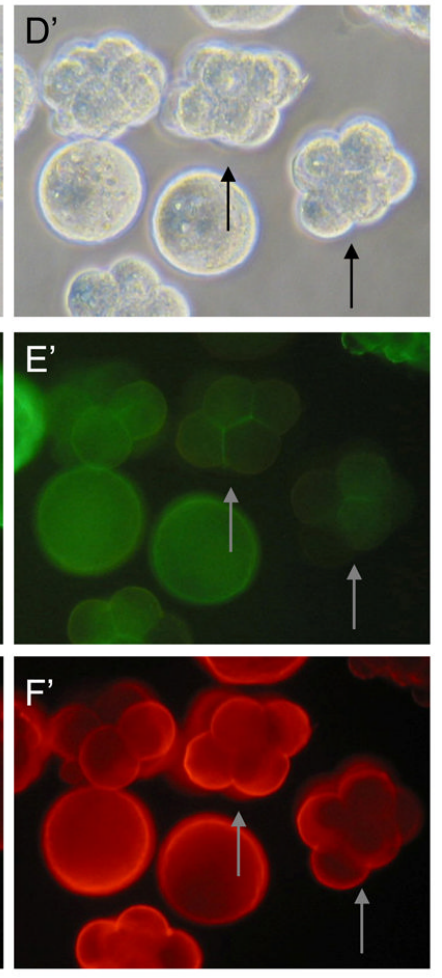

Fig. 6.

SSEA-4 expression on embryos from $T$-syn mutant females. SSEA-4-negative controls were late blastocysts that were included with both control and mutant embryo groups (dashed arrows). A. Phase contrast of control embryos obtained from $T$-syn ${ }^{\mathrm{F} / \mathrm{F}}$ females mated to $T$ $\mathrm{syn}^{+/-}$males. B. Control embryos from A binding PNA. C. Control embryos from A binding the SSEA-4 MAb. Only the early embryos fluoresced brightly. D. Phase contrast of embryos and blastocysts obtained from $T$-syn ${ }^{\mathrm{F} / \mathrm{F}}: \mathrm{ZP} 3 \mathrm{Cre}$ mutant females mated with $T$-syn ${ }^{+/-}$males. E. The same $T$-syn ${ }^{+/-}$and $T$-syn ${ }^{-/-}$embryos from D binding PNA. Early PNA-negative $T$ syn $^{-/-}$embryos are circled. F. The same $T-$ syn $^{+/-}$and $T-$ syn $^{-/-}$embryos from D binding the SSEA-4 MAb. PNA-negative embryos are circled in panels $E$ and $F$ and identified by solid arrows in panels $E^{\prime}$ and $F^{\prime}$. $A^{\prime}-F^{\prime}$. The area marked by each dotted square in A-F is magnified. Images are at the same magnification and the same exposure. Solid arrows point to PNAnegative early embryos. Images are representative of 3 experiments with 2 eggs and 19, 4-8 cell embryos, half of the total generated by 6 control females and 4 eggs and 22, 4-8 cell embryos, half of the total generated by 6 mutant $T$-syn females. 

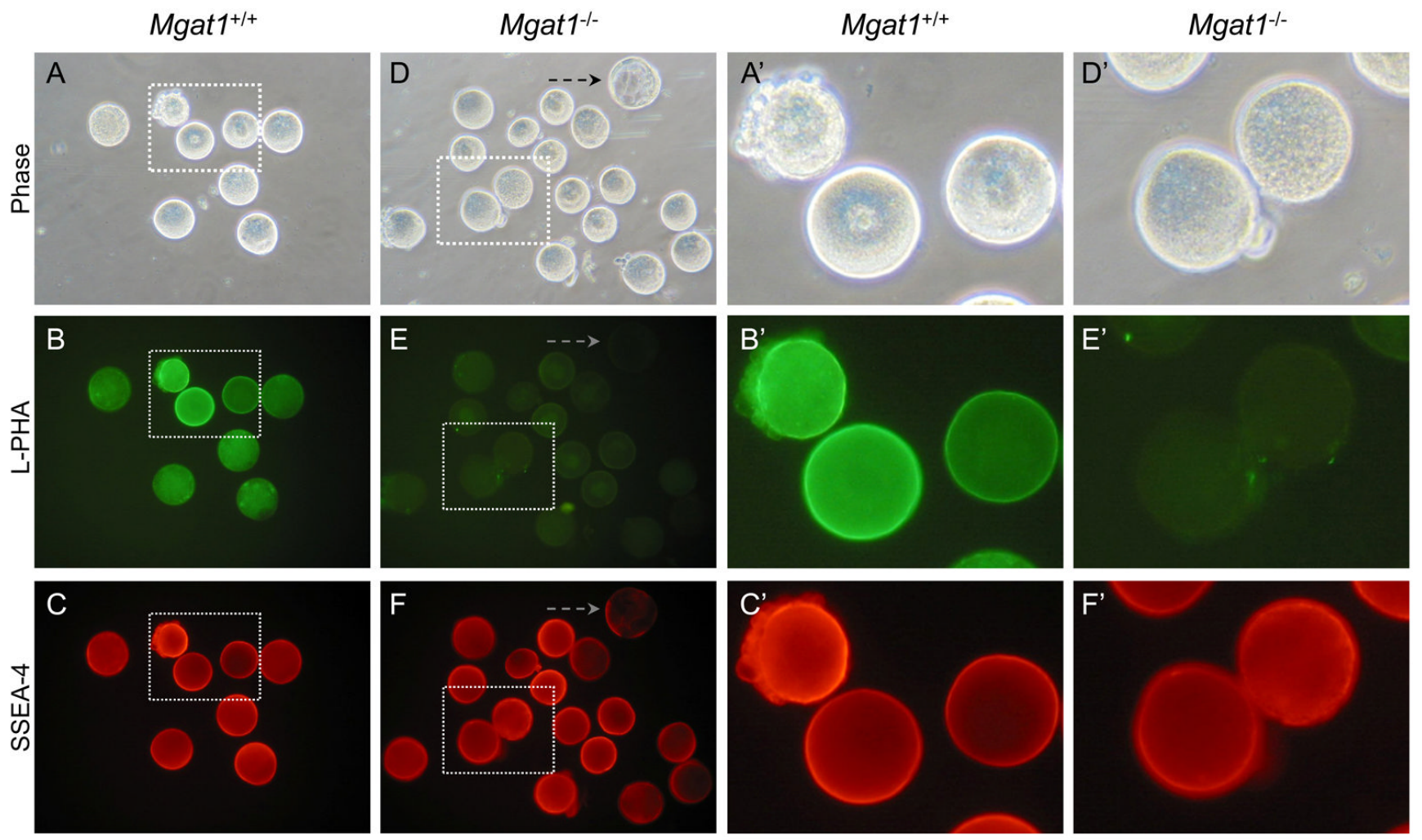

Fig. 7.

SSEA-4 detection on ovulated eggs from Mgatl mutant females. gat $^{\mathrm{FF}}{ }^{\mathrm{ZP}} 3 \mathrm{Cr}$ e females were superovulated and zona-free eggs prepared. A late wild type blastocyst (dashed arrow) was included with the mutant eggs as a negative control for SSEA-4. A. Phase contrast of control zona-free eggs obtained from superovulated $\mathrm{Mgat}^{\mathrm{F} / \mathrm{F}}$ females. B. L-PHA binding to control eggs in A. C. SSEA-4 MAb binding to control eggs in A. D. Phase contrast of zonafree eggs obtained from Mgat $^{\mathrm{F} / \mathrm{F}}: \mathrm{ZP} 3 \mathrm{Cre}$ females. E. L-PHA binding to the Mgatl mutant eggs in D. F. SSEA-4 MAb binding to the mutant eggs in D. L-PHA negative eggs fluoresce strongly. $\mathrm{A}^{\prime}-\mathrm{F}^{\prime}$. The area marked by each dotted square in A-F is magnified. Images are at the same magnification and the same exposure. Images are representative of 2 experiments with 10 eggs and 4 compacted 8 -cell embryos, half of the total generated by 7 control females, and 16 eggs and 6, 4-8 cell embryos, half of the total generated by 5 Mgat 1 mutant females. 


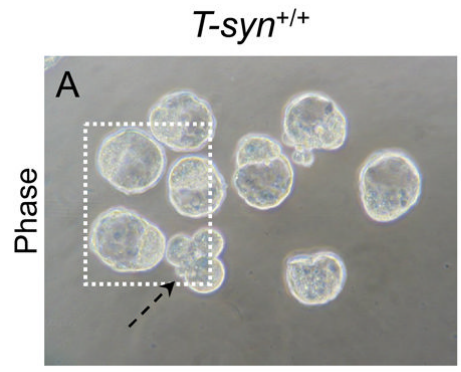

T-syn ${ }^{+/-}$or $T-s y n^{-1-}$
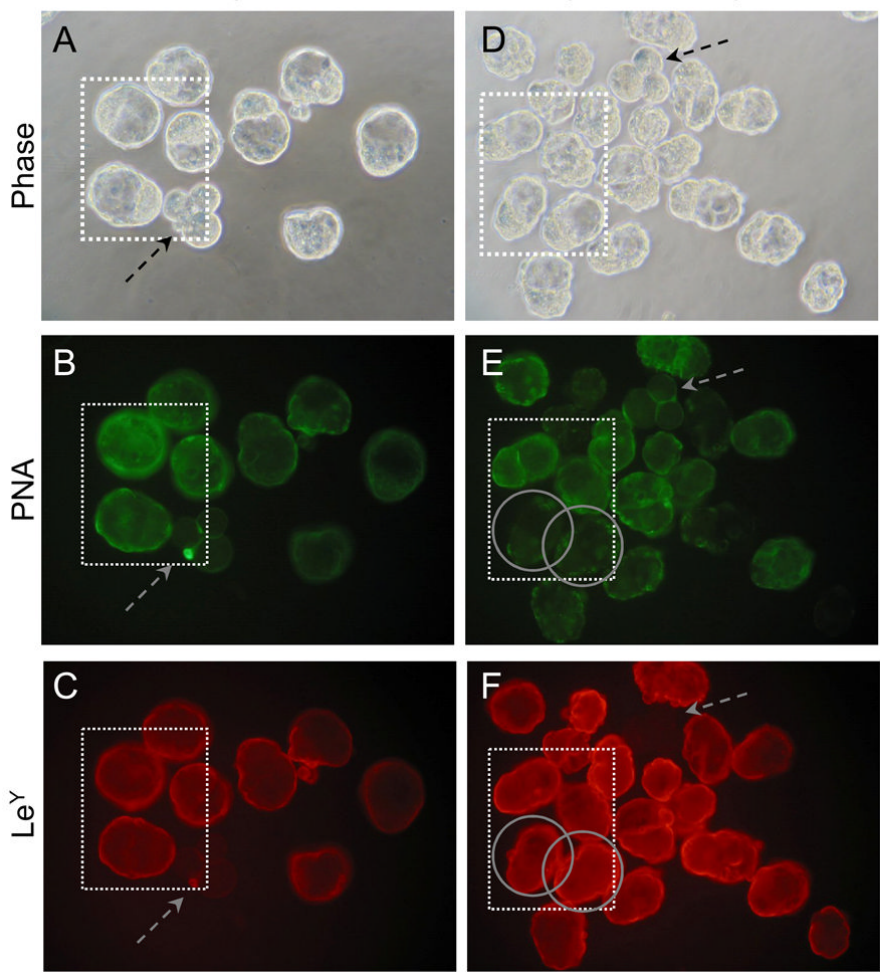
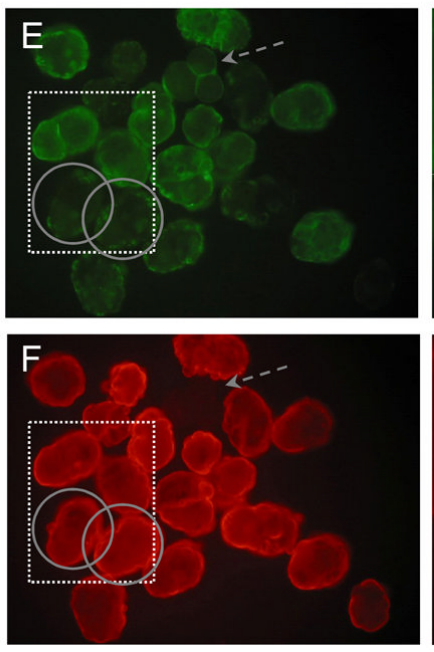

$T-$ syn $^{+/+}$
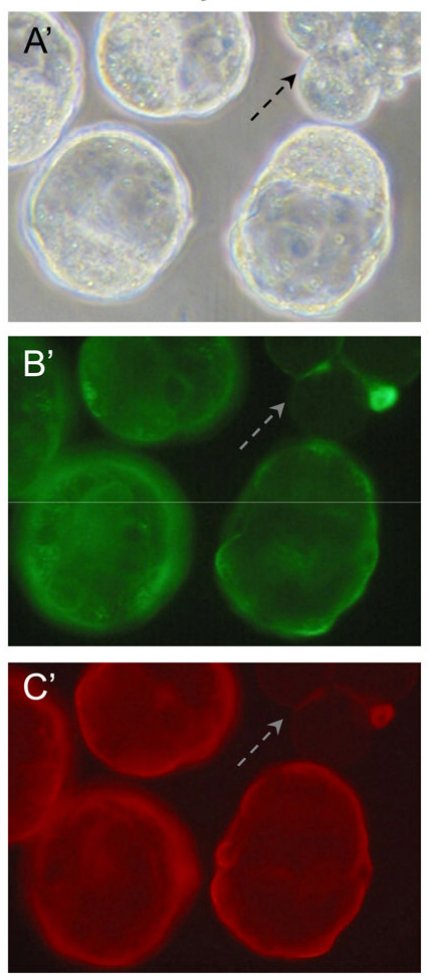

T-syn ${ }^{+/-}$or $T-$ syn $^{-1-}$
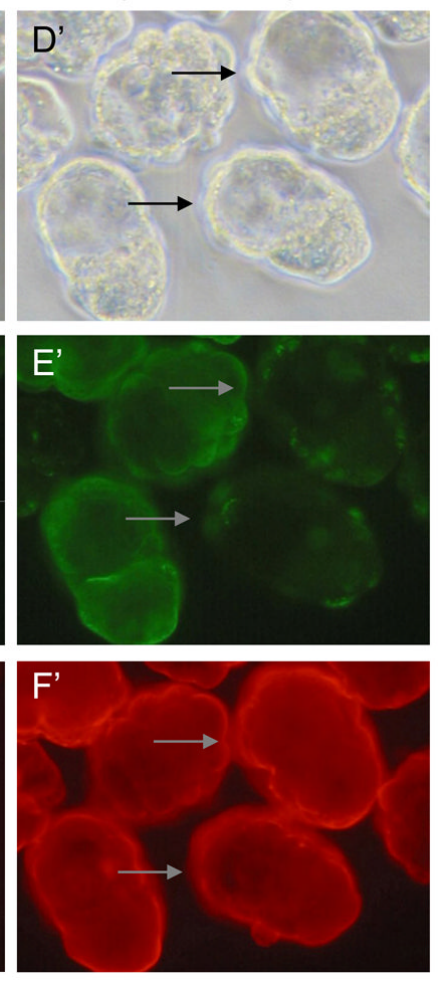

Fig. 8.

$\mathrm{Le}^{\mathrm{Y}}$ expression on blastocysts from $T$-syn mutant females. $\mathrm{Le}^{\mathrm{Y}}$-negative controls were early embryos that were included with both control and mutant embryo groups (dashed arrows). A. Phase contrast of control blastocysts obtained from $T$-syn ${ }^{\mathrm{F} / \mathrm{F}}$ females mated to $T$-syn ${ }^{+/-}$males. B. Control blastocysts from A binding PNA. C. Control blastocysts from A binding the $\mathrm{Le}^{\mathrm{Y}}$ MAb. Only the blastocysts fluoresced brightly. D. Phase contrast of blastocysts obtained from $T$-syn ${ }^{\mathrm{F} / \mathrm{F}}: \mathrm{ZP} 3 \mathrm{Cre}$ mutant females mated with $T$-syn ${ }^{+/-}$males. E. The same $T$-syn ${ }^{+/-}$and $T$ $\operatorname{syn}^{-/-}$blastocysts from D binding PNA. Two PNA-negative $T-s y n^{-/-}$blastocysts are circled. F. The same $T$-syn $n^{+-}$and $T$-syn ${ }^{-l-}$ blastocysts from D binding the Le ${ }^{\mathrm{Y}}$ MAb. PNA-negative blastocysts identified by solid arrows. $A^{\prime}-F^{\prime}$. The area marked by each dotted square in A-F is magnified. Images are at the same magnification and the same exposure and are rotated where necessary. Images are representative of 2 experiments with 10 blastocysts from 2 control females and 30 blastocysts from $4 T$-syn mutant females. 


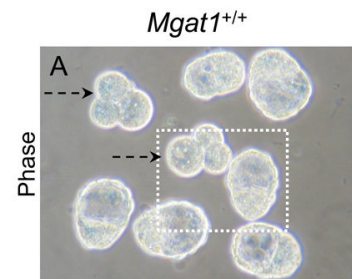

Mgat1+/- or Mgat1-/-
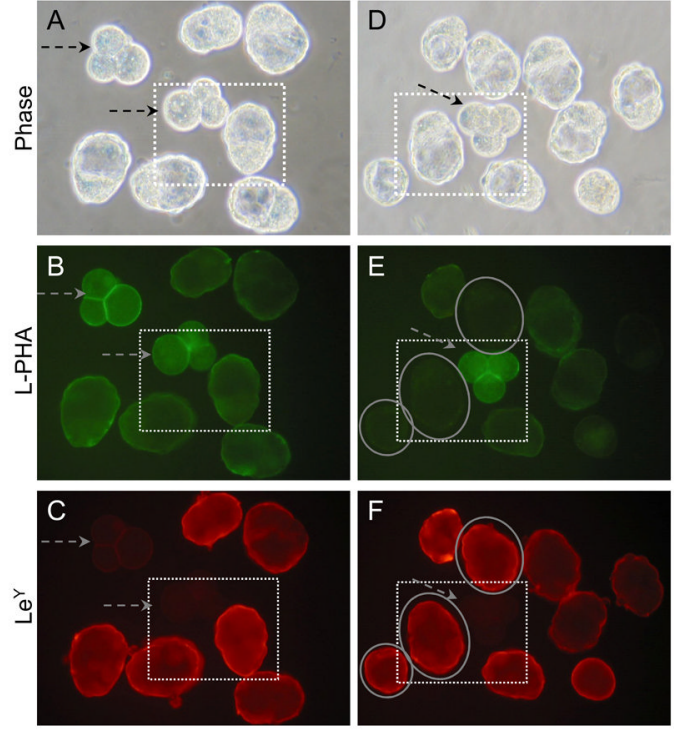

$\mathrm{E}$
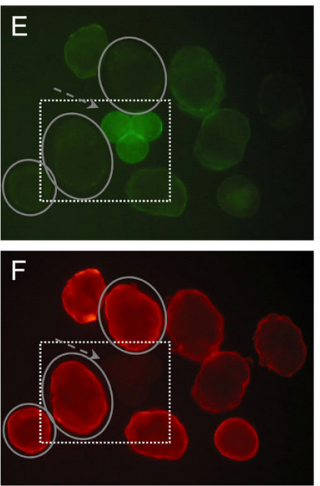

Mgat1 $^{+/+}$
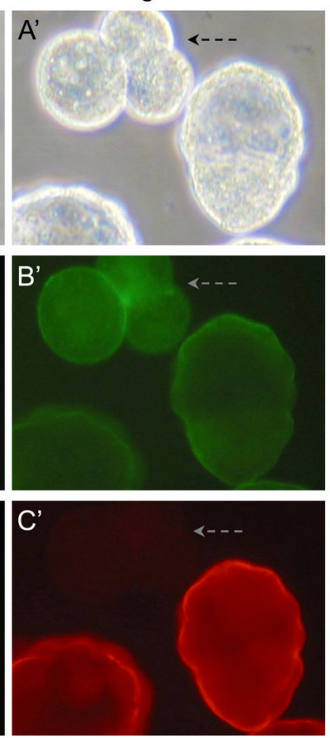

Mgat1+/- or Mgat1-/-
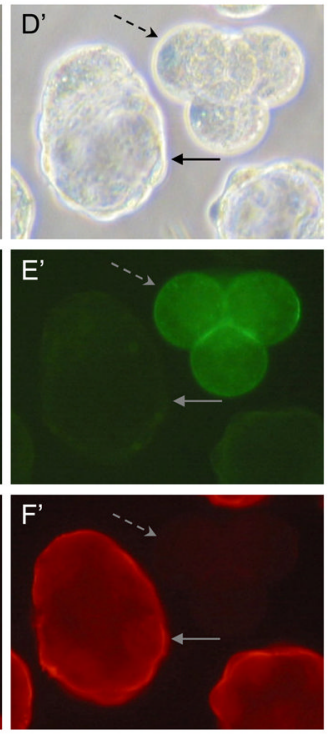

Fig. 9.

$\mathrm{Le}^{\mathrm{Y}}$ expression on blastocysts from Mgatl mutant females. Early embryos were included as $\mathrm{Le}^{\mathrm{Y}}$-negative controls (dashed arrows). A. Phase contrast of control blastocysts obtained from Mgat $^{\mathrm{F} / \mathrm{F}}$ females mated to $\mathrm{Mgatl}^{+/-}$males. B. L-PHA binding to control blastocysts. C. $\mathrm{Le}^{\mathrm{Y}} \mathrm{MAb}$ binding to control blastocysts. D. Phase contrast of blastocysts obtained from Mgat $^{\mathrm{F} / \mathrm{F}}: \mathrm{ZP} 3 \mathrm{Cre}$ females mated to Mgat $^{+/-}$males. E. L-PHA binding to the Mgat1 mutant blastocysts in D. Three L-PHA-negative blastocysts are circled. F. Le ${ }^{\mathrm{Y}} \mathrm{MAb}$ binding to the Mgat1 mutant blastocysts in D. L-PHA negative blastocysts (circled) fluoresce strongly. A'-F '. The area marked by each dotted square in A-F. Images are at the same magnification and the same exposure. Images are from one experiment due to the low fertility of $\mathrm{Mgat}^{\mathrm{F} / \mathrm{F}}: \mathrm{ZP} 3 \mathrm{Cre}$ females. 